Document downloaded from:

http://hdl.handle.net/10251/78055

This paper must be cited as:

Feliu-Pérez, J.; Sahuquillo Borrás, J.; Petit Martí, SV.; Duato Marín, JF. (2014). Addressing bandwidth contention in SMT multicores through scheduling. ACM.

doi:10.1145/2597652.2600109.

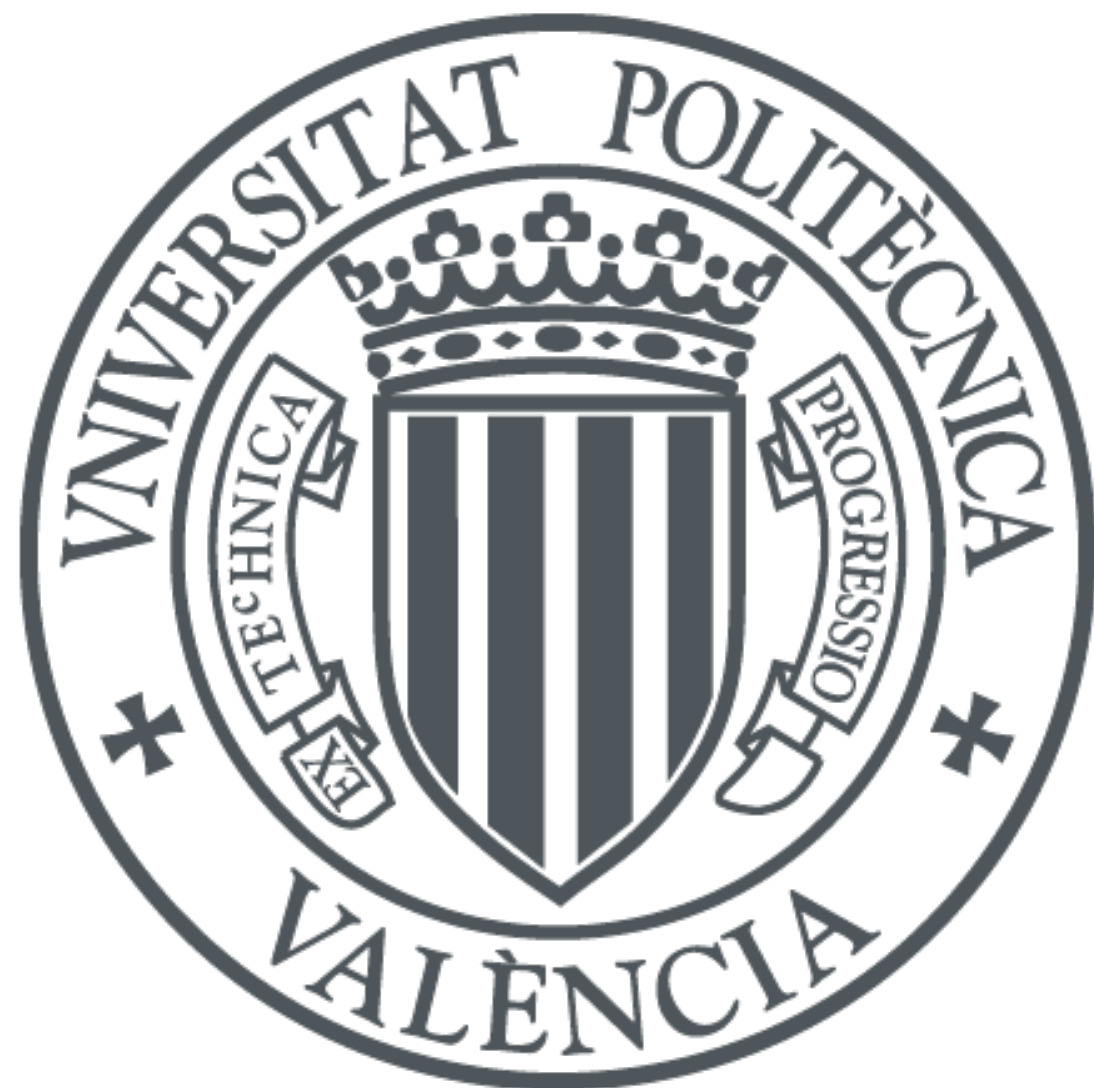

The final publication is available at

http://dl.acm.org/citation.cfm?id=2600109

Copyright ACM

Additional Information

(C) Owner/Author 2014. This is the author's version of the work. It is posted here for your personal use. Not for redistribution. The definitive Version of Record was published in ICS '14 Proceedings of the 28th ACM international conference on Supercomputing; http://dx.doi.org/10.1145/2597652.2600109 


\title{
Addressing Bandwidth Contention in SMT Multicores Through Scheduling
}

\author{
Josué Feliu, Julio Sahuquillo, Salvador Petit, and José Duato \\ Department of Computer Engineering \\ Universitat Politècnica de València \\ València, Spain \\ jofepre@gap.upv.es, \{jsahuqui,spetit,jduato\}@disca.upv.es
}

\begin{abstract}
To mitigate the impact of bandwidth contention, which in some processes can yield to performance degradations up to $40 \%$, we devise a scheduling algorithm that tackles main memory and L1 bandwidth contention. Experimental evaluation on a real system shows that the proposal achieves an average speedup by $5 \%$ with respect to Linux.
\end{abstract}

\section{Categories and Subject Descriptors}

D.4.1 [Operating Systems]: Process management—Scheduling Keywords

bandwidth-aware scheduling; bandwidth contention

\section{PROPOSED SCHEDULER}

Algorithm 1 presents the pseudocode of the devised scheduler. It consists of process selection (lines 2-8) and process allocation (lines 9-12), which deal with main memory and L1 bandwidth contention, respectively, by balancing the memory requests over the workload execution time and the L1 requests among the L1 caches. Previously, the scheduler calculates the average main memory transaction rate of the workload following a similar approach to [2]

In the process selection, the proper set of processes is selected to be run during the following quantum. The process not executed for longer is always selected to avoid process starvation. Then, the remaining processes are selected using the fitness function, which quantifies the gap between the $\mathrm{TR}_{M M}$ required by a given process and the average bandwidth remaining for each unallocated hardware thread [2].

In the process allocation, the selected processes are allocated to the cores. Since the experimental platform implements dual-threaded cores, the L1 bandwidth can be easily balanced by sorting the processes according to its $\mathrm{TR}_{L 1}$ and then, reiteratively, assigning the processes with highest and lowest bandwidth utilization to the same core [1].

\section{EXPERIMENTAL EVALUATION}

The experimental evaluation is carried out in an Intel Xeon E5645 processor, with six dual-thread SMT cores, a

Permission to make digital or hard copies of part or all of this work for personal or classroom use is granted without fee provided that copies are not made or distributed for profit or commercial advantage, and that copies bear this notice and the full citation on the first page. Copyrights for third-party components of this work must be honored. For all other uses, contact the owner/author(s). Copyright is held by the author/owner(s).

ICS'14, June 10-13 2014, Munich, Germany.

ACM 978-1-4503-2642-1/14/06.

http://dx.doi.org/10.1145/2597652.2600109.
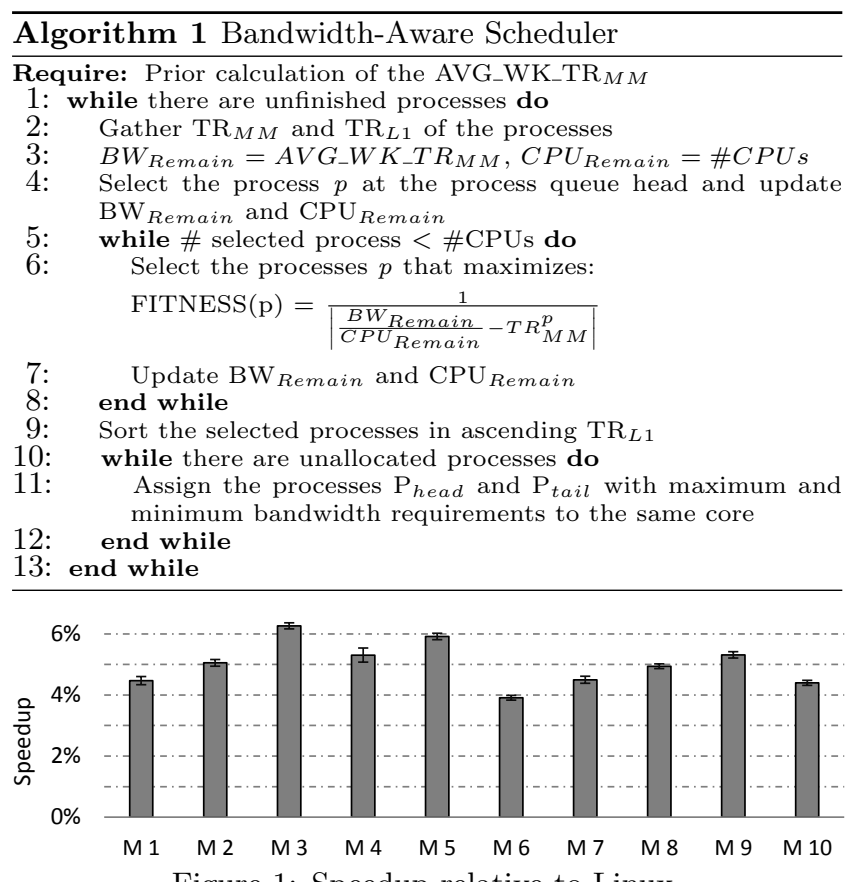

Figure 1: Speedup relative to Linux.

private L1 cache per core and a shared LLC. The algorithm has been implemented in a user-level scheduler. To evaluate the performance of the proposal, a set of ten 24-benchmark mixes was designed.

Figure 1 presents the speedup the devised scheduler achieves compared to the Linux scheduler across all the mixes using the average IPC with $95 \%$ confidence intervals. Results show that the scheduler effectively addresses bandwidth contention and improves the Linux performance by $5 \%$ on average.

\section{ACKNOWLEDGMENTS}

This work was supported by the Spanish Ministerio de Economía y Competitividad (MINECO) and Plan E funds, under Grant TIN2012-38341-C04-01, and by the Intel Early Career Faculty Honor Program Award.

\section{REFERENCES}

[1] J. Feliu, J. Sahuquillo, S. Petit, and J. Duato. L1-Bandwidth Aware Thread Allocation in Multicore SMT Processors. In PACT, pages 123-132, 2013.

[2] D. Xu, C. Wu, and P.-C. Yew. On mitigating memory bandwidth contention through bandwidth-aware scheduling. In PACT, pages 237-248, 2010. 